\title{
Deep Learning for Atomically Resolved Imaging
}

Maxim Ziatdinov ${ }^{1,2}$, Ondrej Dyck ${ }^{1,2}$, Stephen Jesse ${ }^{1,2}$ and Sergei V. Kalinin ${ }^{1,2}$

1. Center for Nanophase Materials Sciences, Oak Ridge National Laboratory, Oak Ridge TN.

2. Institute for Functional Imaging of Materials, Oak Ridge National Laboratory, Oak Ridge TN.

Recent advances in scanning transmission electron microscopy (STEM) and scanning tunneling microscopy (STM) allow unprecedented opportunities in probing the materials structural parameters and electronic properties in real space with an angstrom-level precision. These experimental capabilities require development of tools for the rapid, physics-guided analysis of the very large amount of data generated by modern day microscopes, ideally in a real-time. Here we argue that one of the most promising methods for creating such an AI-powered microscope is based on deep neural networks [1,2].

We first define topology of a deep learning (DL) model for atomic imaging. Because it is crucial to locate accurately all the atomic species and point defects in the image, the model must ideally provide a pixel-wise classification map of the same size as the input image. One possible candidate is the classactivation-maps-based DL analysis, in which a model trained on image-level labels is capable in principle of discriminating the image regions used to identify the specific class [3]. The disadvantage of such approach is that while it may correctly identify the location and type of certain defects, it doesn't allow finding all the atoms and/or atomic columns in the data. The alternative approach that we chose here is to use a fully convolutional neural network model, which can be trained to output a pixel-wise classification map, with the same size/resolution as the original input image, that shows a probability of each pixel belonging to certain type of atom, atomic column and/or defect.

Another critical aspect is the creation of an appropriate training set. We show that it is possible to use the deep convolutional neural network trained on the theoretical STEM data created using Multislice algorithm to obtain accurate pixel-wise classification maps for the experimental STEM data. We were able to train DL models specific for each lattice type (by enforcing a periodicity specific to the lattice) as well as a single DL model capable of finding the atoms / atomic columns in lattices with different symmetry (by removing hard periodic constraints).

It is quite common that apriori information about details of various atomic and defect configurations in the materials of interest is very limited. To solve this issue, we developed a "weakly-supervised" DL approach for the analysis of experimental data on 2-dimensional materials [1]. Specifically, we start with "knowing" only a limited number of defect classes and then use information on the coordinates of all atomic species in the image extracted via a DL model, combined with basic chemistry rules introduced via a graph representation, to identify a rich variety of defects that are not part of an initial training set.

One interesting aspect of working with deep convolutional neural networks for atomic imaging is that it is possible to perform segmentation of individual atoms and atomic columns for the noisy, un-processed experimental data [2]. In this case, the geometrical descriptors of the segmented 'atomic contours' can be linked to certain physical and chemical processes in the materials. We used this approach to find anomalies in the lattice structure of 2-d materials as well as to analyze transformations in 3-d samples of Si under e-beam irradiation. 
Unlike the STEM image, which primarily reveals atomic positions, the STM image is associated with the electronic density of states around the Fermi level. The automatic analysis of STM images is notoriously difficult compared to STEM, reflecting the more delocalized nature of STM signal. As a result, image analytical methods developed for STEM have rarely been applied to STM. However, our DL model trained to extract segmented 'atomic contours' in STEM images could easily overcome these obstacles allowing us to analyze in the automated fashion peculiarities of the charge density distribution on the surface of manganites and positional and orientational orders in the self-assembly of buckyballs on gold [4].

\section{References:}

[1] M. Ziatdinov et al, ACS Nano 11 (2017), p. 12742.

[2] M. Ziatdinov et al, arXiv:1801.05133 (2018).

[3] B. Zhou, et al, arXiv:1512.04150 (2015).

[4] The work was supported by the U.S. Department of Energy, Office of Science, Materials Sciences and Engineering Division (M.Z., S.V.K.). This research was conducted and partially supported (O. D., S.J.) at the Center for Nanophase Materials Sciences, which is a US DOE Office of Science User Facility.

(a)
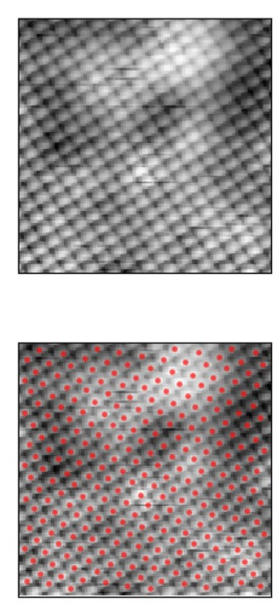

(b)

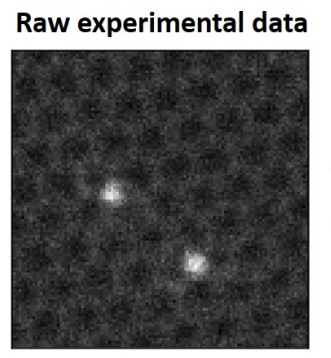

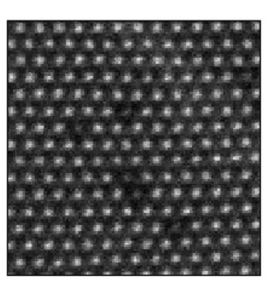

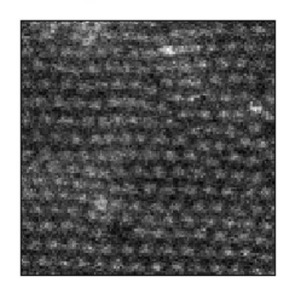

DL output (coordinates)
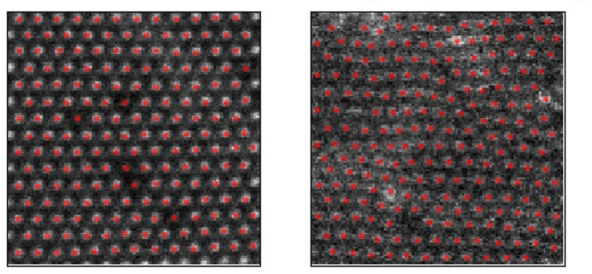
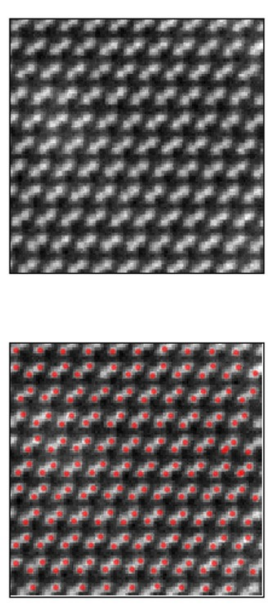
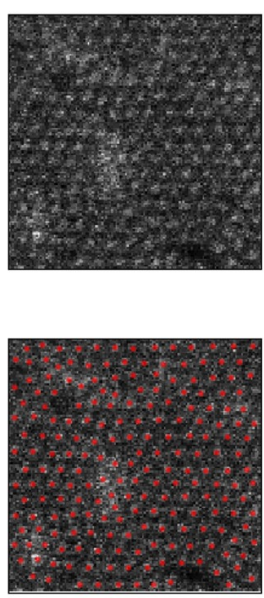

(c)

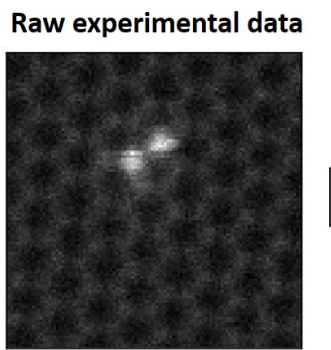

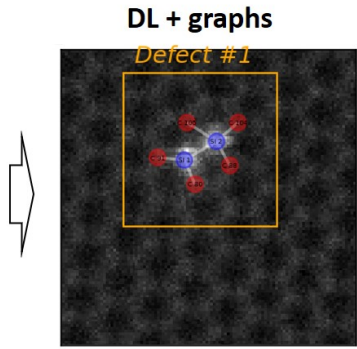

Figure 1. (a) Using a single deep learning model to identify positions of atoms and atomic columns in the images of different materials obtained via STM (1 ${ }^{\text {st }}$ column) and STEM (columns 2-5) experiments. (b-c) STEM images of graphene and identified positions and type of atomic species for the extracted single defect structures. 